\title{
Artigo Original \\ ANÁLISE DE FRATURAS DIAFISÁRIAS DO FÊMUR EM CRIANÇAS MENORES DE 3 ANOS DE IDADE
}

\section{FEMORAL DIAPHYSEAL FRACTURES: AN ASSESSMENT IN CHILDREN YOUNGER THAN 3 YEARS OLD}

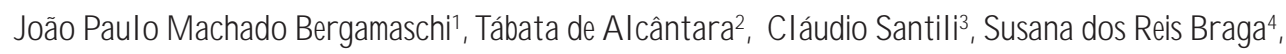 \\ Gill ber to W aisber ${ }^{5}$, M iguel A kkari ${ }^{6}$
}

\begin{abstract}
RESUMO
Este estudo consiste na reavaliação ortopédica e psicossocial de crianças que sofreram fratura do fêmur até a idade dos três anos e objetiva a análise de suas causas prováveis e detecção de indícios de ocorrência de Síndrome de Maus Tratos. Trinta e cinco crianças menores de três anos de idade sofreram fratura diafisária de fêmur e foram atendidas no Pronto Socorro do Serviço de Ortopedia e Traumatologia da Santa Casa de São Paulo, no período de janeiro de 1996 a agosto de 2002, sendo que 18 compareceram para reavaliação. Como causa relatada das fraturas observamos: queda em $13(72,2 \%)$ casos, queda de objetos sobre o membro em três $(16,7 \%)$ e fratura no parto em dois $(11,1 \%)$. Constatamos suspeita de Síndrome dos Maus Tratos em nove (maus tratos físicos em seis [33,3\%] e negligência em três [16,7\%]) casos, fratura patológica em quatro (22,2\%), causa acidental em três $(16,7 \%)$, e outras causas em dois $(11,1 \%)$ casos. Maus tratos constituem uma importante causa a ser investigada nos casos de fratura do fêmur em crianças com menos de três anos de idade, sendo o provável mecanismo responsável por metade das fraturas aqui estudadas.
\end{abstract}

Descritores: Fratura de fêmur, Maus tratos, Crianças.

Citação: Bergamaschi JPM, Alcântara T, Braga SR, Akkari M, Santili C. Análise de fraturas diafisárias do fêmur em crianças menores de 3 anos de idade. Acta Ortop Bras. [periódico na Internet]. 2007; 15(2): 72-75. Disponível em URL: http://www. scielo.br/aob.

\section{INTRODUÇÃO}

Caffey $^{(1)}$, em 1946, foi o primeiro a relacionar as fraturas de ossos longos com hematoma subdural crônico, mas o mecanismo de trauma de tais lesões ainda era desconhecido. Somente em 1962, foi utilizado pela primeira vez o termo "Síndrome da criança espancada", por Kempe et al (2), para descrever uma condição clínica na criança vítima de maus tratos físicos.

A fratura de ossos longos em crianças de pouca idade pode representar uma das principais evidências de alto grau de maus tratos físicos ${ }^{(3)}$, sendo que a fratura do fêmur está associada à Síndrome de Maus Tratos (SMT) em 30-36\% dos casos que acometem crianças menores de três anos de idade ${ }^{(4)}$, e em até $85 \%$ em crianças com menos de um ano de idade ${ }^{(5,6)}$.

Identificar e caracterizar a condição de mau trato é difícil em muitos casos ${ }^{(2,5,6)}$ e baseia-se a suspeita mediante sinais diretos (sinais e sintomas) e indiretos (histórico da lesão), pois raramente há confissão do agressor. A não identificação pode favorecer a recidiva da agressão e caso a criança retorne ao ambiente domiciliar pode ser vítima de seqüelas psicomotoras graves ou mesmo de morte ${ }^{(7,8,9)}$.

\section{SUMMARY}

This study consists of an orthopaedic and psychosocial re-evaluation of children who experienced femur fractures as young as 3 years old and aims to analyze potential causes and detect Child Abuse rates. Thirty-five children under the age of three years who experienced femoral diaphyseal fractures received care at the Emergency Department of the Orthopaedics and Traumatology Service of Santa Casa de São Paulo within the period ranging from January, 1996 to August, 2002. Eighteen patients returned to the hospital for re-evaluation. The reported causes for fractures were: fall in 13 cases (72.2\%), object fall on the limb in 2 cases $(11.1 \%)$. Child abuse was suspected in 9 cases (physical abuse in 6 cases $(33.3 \%)$, negligence in 3 cases $(16.7 \%)$, pathological fracture in 4 cases $(22.2 \%)$, accidental causes in 3 cases $(16.7 \%)$, and other causes in 2 cases (11.1\%). Child abuse constitutes an important cause that needs to be investigated in cases of femur fracture in children under the age of three years and this has possibly been the mechanism responsible for half of the fractures investigated in the current study.

Keywords: Femoral fractures, Child abuse, Children.

Citation: Bergamaschi JPM, Alcântara T, Braga SR, Akkari M, Santili C. Femoral diaphyseal fractures: an assessment in children younger than 3 years old. Acta Ortop Bras. [serial on the Internet]. 2007; 15(2):72-75. Available from URL: http://www.scielo.br/aob.

Este estudo retrospectivo compreende a reavaliação ortopédica e psicossocial de crianças que sofreram fratura do fêmur até a idade dos três anos e objetiva a análise de suas causas prováveis e detecção de indícios de ocorrência de SMT.

\section{PACIENTES E MÉTODOS}

Trinta e cinco crianças menores de três anos de idade sofreram fratura diafisária de fêmur e foram atendidas no Pronto Socorro do Serviço de Ortopedia e Traumatologia da Santa Casa de São Paulo, no período de janeiro de 1996 a agosto de 2002. Dezenove crianças e seus respectivos responsáveis foram contatados através de telefonemas e cartas enviadas aos endereços coletados no prontuário médico, sendo que 18 compareceram para reavaliação. O projeto foi, inicialmente, aprovado pelo Comitê de Ética em Pesquisa da Irmandade da Santa Casa de Misericórdia de São Paulo (Protocolo n²42/02). Foram recoletados dados de anamnese relacionados à história da fratura e de eventuais outras fraturas ou internações: tempo decorrido do momento da fratura até o atendimento médico; história ou presença de sinais de negligência e investigação sobre as condições sócio-econômicas da família e/ou responsáveis pela

Instituição: Departamento de Ortopedia e Traumatologia da Santa Casa de Misericórdia de São Paulo

Endereço para correspondência: Rua Cesário Motta Júnior, 112 - Vila Buarque - São Paulo / SP - Departamento de Ortopedia e Traumato
- 50 andar - E-mail: c.santili@terra.com.br; jberga@uol.com.br
1- Médico Residente em Ortopedia e Traumatologia da Santa Casa de Misericórdia de São Paulo
2- Médica Ortopedista e Ex-Estagiária do Grupo de Ortopedia e Traumatologia Pediátrica da Santa Casa de Misericórdia de São Paulo
3- Diretor do Departamento de Ortopedia e Traumatologia da Santa Casa de Misericórdia de São Paulo
4- Médica Assistente do Grupo de Ortopedia e Traumatologia Pediátrica da Santa Casa de Misericórdia de São Paulo
5- Médico Assistente do Grupo de Ortopedia e Traumatologia Pediátrica da Santa Casa de Misericórdia de São Paulo
6- Chefe do Grupo de Ortopedia e Traumatologia Pediátrica da Santa Casa de Misericórdia de Sáo Paulo
Trabalho recebido em 29/06/06 e aprovado em 01/09/06 
criança, através de protocolo previamente elaborado. O exame físico consistiu da mensuração clínica dos membros inferiores. Como complementos foram solicitadas radiografias do membro acometido (incidências ântero-posterior e perfil) e escanometria dos membros inferiores para verificar a consolidação da fratura e/ou alterações no crescimento do membro fraturado. Os pacientes e seus responsáveis foram ainda submetidos à avaliação psicológica individual, realizada por duas psicólogas do Serviço de Psicologia da Santa Casa de São Paulo, visando identificar relatos discordantes e/ou fatores suspeitos de maus tratos físicos, como: má estrutura familiar, história prévia de abuso na família, gravidez não desejável, vínculo afetivo pobre entre pai/mãe X filho(a), uso de drogas ilícitas, alcoolismo, baixa escolaridade, desemprego, doenças psiquiátricas, distúrbios emocionais e de personalidade. Foi realizada discussão detalhada de cada caso reavaliado, relacionando informações do prontuário médico com os dados coletados na reavaliação ortopédica e psicológica, a fim de identificar o provável mecanismo de ocorrência das fraturas.

Para classificação da causa da fratura, utilizamos a escala de Thomas et al modificada $(5,6)$ :

1. Abuso físico confirmado: confissão por parte da criança ou pais e/ou responsáveis; presença de múltiplas fraturas não condizentes com a história relatada; presença de sinais e sintomas como, equimoses, queimaduras, hematomas e escoriações não explicados, presença de alterações comportamentais graves.

2. Suspeita de abuso físico: histórias discordantes contadas por diferentes pessoas; histórias inconsistentes e não suficientes para a ocorrência da lesão, demora na procura de atendimento médico após a ocorrência da lesão, presença de alterações comportamentais, afastamento da criança do ambiente familiar e/ou de algum membro da família.

3. Negligência: falta de supervisão e cuidados com a criança, levando a lesões que poderiam ser evitadas.

4. Causa desconhecida: história incompleta e não suspeita, informações insuficientes.

5. Lesão patológica: presença de doença de base (osteogenese imperfeita, neoplasia, etc), com suspeita ou confirmação de abuso físico descartadas.

6. Acidente: fratura não suspeita, história consistente e completa, história comprovada.

7. Outros: falta de orientação dos cuidados básicos com a criança por parte dos responsáveis, sem suspeita ou confirmação de SMT. Realizamos ainda uma busca para identificar o motivo do não comparecimento ou da impossibilidade de contato dos pacientes que não estiveram presentes na reavaliação $(n=1)$ ou não foram contatados $(n=17)$ através dos dados pessoais, telefonemas e endereços contidos no prontuário médico.

\section{RESULTADOS}

A idade média no momento da fratura dos 18 pacientes reavaliados foi de 20,0 \pm 10,9 (0-34) meses (Gráfico 1).

Houve uma predominância do sexo masculino $(77,8 \%)$ entre as crianças reavaliadas (Masc: 14, Fem: 4). Não houve predomínio em relação ao lado de ocorrência da fratura $(D=10, E=8)$. 0 tempo de seguimento médio foi de 56,0 \pm 21,7 meses (24-104 meses) sendo que no momento da reavaliação, os pacientes apresentaram uma idade média de 76,1 \pm 23,1 meses (34 - 133 meses). A procura por atendimento médico ocorreu no mesmo dia da lesão em $12(66,7 \%)$ casos, após um dia da lesão em três $(16,7 \%)$ casos, após dois dias em dois $(11,1 \%)$, e após um mês em um $(5,5 \%)$ caso.

Como causa relatada das fraturas observamos: queda em 13 (72,2\%) casos, queda de objetos sobre o membro em três (16,7\%) e fratura no parto em dois $(11,1 \%)$. Entre os 13 pacientes cuja causa relatada da fratura foi a queda, observamos relato de queda da própria altura em três casos, queda da cama ou colchão em dois, queda do colo da mãe em dois, queda da cadeira em um, queda da escada em

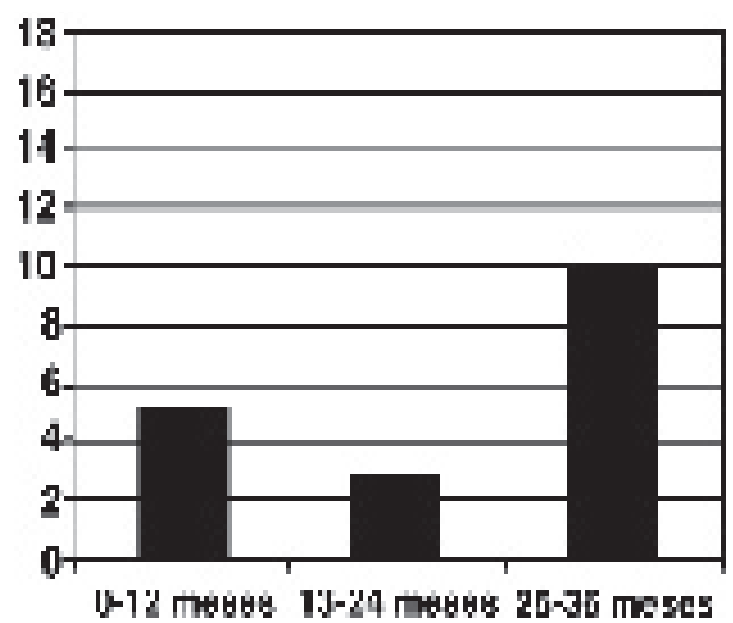

Fonte: SAME do Departamento de Ortopedia e Traumatologia da Santa Casa de Misericórdia de São Paulo.

Gráfico 1 - Distribuição dos 18 pacientes quanto à idade.

quatro e queda de laje em um caso (Tabela 1). Após a reavaliação ortopédica e psicológica, constatamos suspeita de maus tratos físicos em seis $(33,3 \%)$ casos, negligência em três (16,7\%), fratura patológica em quatro (22,2\%), causa acidental em três (16,7\%), e outras causas em dois $(11,1 \%)$ casos (Gráfico 2, Tabela 1). Das quatro crianças com fratura em osso patológico (três por osteogenese imperfeita e um por fêmur curto congênito), três haviam apresentado episódios anteriores de fratura do fêmur: um paciente sofrera três fraturas e dois apresentaram outras duas.

O perfil social dos pacientes e seus familiares estão relacionados na Tabela 2. Gravidez não desejada foi relatada em um caso. Encon-

\begin{tabular}{|c|c|c|c|}
\hline$n$ & $\begin{array}{l}\text { Causa } \\
\text { relatada }\end{array}$ & $\begin{array}{l}\text { Causa } \\
\text { Constatada }\end{array}$ & $\begin{array}{l}\text { Causa } \\
\text { Escala }\end{array}$ \\
\hline 1 & fratura no parto & indeterminada & outros \\
\hline 2 & queda no banho & indeterminada & outros \\
\hline 3 & $\begin{array}{l}\text { queda do colo da mãe } \\
\text { na rua }\end{array}$ & queda do colo & acidente \\
\hline 4 & pulou da laje & queda da laje & negligência \\
\hline 5 & $\begin{array}{l}\text { escorregou e a tábua } \\
\text { de passar roupa caiu sobre } \\
\text { sobre o membro }\end{array}$ & $\begin{array}{l}\text { queda de objeto } \\
\text { o membro }\end{array}$ & acidente \\
\hline 6 & $\begin{array}{l}\text { escorregão em } \\
\text { rampa molhada }\end{array}$ & $\begin{array}{l}\text { queda da } \\
\text { própria altura }\end{array}$ & negligência \\
\hline 7 & $\begin{array}{l}\text { queda da cama sobre a perna } \\
\text { enquanto brincava com o tio }\end{array}$ & jogado da cama & $\begin{array}{l}\text { suspeita de maus } \\
\text { tratos físicos }\end{array}$ \\
\hline 8 & queda da cadeira & queda da cadeira & lesão patológica \\
\hline 9 & fratura no parto & fratura no parto & lesão patológica \\
\hline 10 & torção ao pular no colchão & queda da própria altura & lesão patológica \\
\hline$\overline{11}$ & queda da escada $(h=1 \mathrm{~m})$ & queda da escada & $\begin{array}{l}\text { suspeita de maus } \\
\text { tratos físicos }\end{array}$ \\
\hline$\overline{12}$ & queda ao subir um degrau & $\begin{array}{l}\text { queda da } \\
\text { própria altura }\end{array}$ & $\begin{array}{l}\text { suspeita de maus } \\
\text { tratos físicos }\end{array}$ \\
\hline 13 & queda da escada $(h=1 \mathrm{~m})$ & queda da escada & negligência \\
\hline 14 & queda do colo da mãe & queda da laje & $\begin{array}{l}\text { suspeita de maus } \\
\text { tratos físicos }\end{array}$ \\
\hline 15 & queda na creche & queda da própria altura & $\begin{array}{l}\text { suspeita de maus } \\
\text { tratos físicos }\end{array}$ \\
\hline 16 & $\begin{array}{l}\text { queda da cadeira e cadeira } \\
\text { caiu sobre o membro }\end{array}$ & atropelamento & $\begin{array}{l}\text { suspeita de maus } \\
\text { tratos físicos }\end{array}$ \\
\hline 17 & $\begin{array}{l}\text { queda da escada e escada } \\
\text { caiu sobre o membro }\end{array}$ & $\begin{array}{l}\text { queda de objeto } \\
\text { sobre o membro }\end{array}$ & acidente \\
\hline 18 & queda da escada & queda da escada & lesão patológica \\
\hline
\end{tabular}

Fonte: SAME do Departamento de Ortopedia e Traumatologia da Santa Casa de Misericórdia de São Paulo.

Tabela 1- Tabela comparativa quanto às causas da fratura nas 18 crianças conforme a causa relatada, a causa constatada e a causa segundo critérios da escala modificada. 


\begin{tabular}{|c|c|c|c|c|c|c|c|c|c|c|c|c|}
\hline & $\begin{array}{l}\text { Situação } \\
\text { Pais }^{A}\end{array}$ & $\begin{array}{c}N^{\circ} \\
\text { irmãos }\end{array}$ & Gravidez $^{B}$ & \begin{tabular}{|l} 
Tent. \\
de Abortoc
\end{tabular} & $\begin{array}{l}\text { Antec. } \\
\text { de violência } \\
\text { Familiar }^{d}\end{array}$ & Antec. $P Q$ & $\begin{array}{c}\text { Uso } \\
\text { le drogas }\end{array}$ & Etilismo & \begin{tabular}{|l|}
$\begin{array}{l}\text { Renda familiar } \\
\text { o pessoas } \\
\text { (reais) }{ }^{f}\end{array}$ \\
\end{tabular} & $\begin{array}{l}\text { Escol. } \\
\mathrm{Pai}^{g}\end{array}$ & \begin{tabular}{c|} 
Escol. \\
Mãe \\
$g$
\end{tabular} & $\begin{array}{c}\text { Cond. } \\
\text { Moradia }^{h}\end{array}$ \\
\hline 1 & $D$ & 3 & $D$ & $N$ & $N$ & $N$ & $N$ & $N$ & 150 & $E B$ & $E B$ & OK \\
\hline 2 & $C$ & 2 & $D$ & $N$ & $N$ & $S$ (mãe) & $N$ & $N$ & 175 & EB inc & $E B$ inc & $O K$ \\
\hline 3 & $C$ & 4 & $D$ & $N$ & $N$ & $N$ & $N$ & $N$ & 250 & EB inc & $E B$ inc & $O K$ \\
\hline 4 & C & 2 & $D$ & $N$ & $N$ & $N$ & $N$ & $N$ & 100 & EB inc & $E B$ inc & OK \\
\hline 5 & $C$ & 2 & $D$ & $N$ & $N$ & $N$ & $S$ (pai) & $N$ & 130 & $E B$ inc & $E B$ inc & $O K$ \\
\hline 6 & $D$ & 2 & $D$ & $N$ & $S$ (pai) & $N$ & $N$ & $S$ (pai) & 200 & $E F$ & $E F$ & OK \\
\hline 7 & $D$ & 1 & $D$ & $N$ & $N$ & $N$ & $N$ & $N$ & 500 & $E B$ & $E B$ & OK \\
\hline 8 & $D$ & 2 & $D$ & $N$ & $N$ & $N$ & $N$ & $N$ & 215 & $E B$ & $E B$ & OK \\
\hline 9 & $S$ & 2 & $D$ & $N$ & $N$ & $N$ & $N$ & $S$ (pai) & 250 & $E F$ & $E B$ inc & OK \\
\hline 10 & $D$ & 1 & $D$ & $N$ & $S$ (pai) & $N$ & $N$ & $N$ & 130 & $E B$ inc & $E B$ inc & $O K$ \\
\hline 11 & $C$ & 4 & $D$ & $N$ & $N$ & S(avó) & $S(2$ tios) & $N$ & 86 & $E B$ inc & $E B$ inc & $O K$ \\
\hline 12 & $D$ & 4 & $D$ & $N$ & $S$ (pai) & $N$ & $N$ & $N$ & 200 & $E F$ & $E F$ & $O K$ \\
\hline 13 & $D$ & 3 & $D$ & $N$ & $N$ & $S(m a ̃ e)$ & S (mãe) & $N$ & 240 & $E B$ & $E B$ & OK \\
\hline 14 & $D$ & 2 & $D$ & $N$ & $N$ & $N$ & $N$ & $N$ & 260 & $E B$ & $E B$ & $O K$ \\
\hline 15 & $D$ & 2 & I & $N$ & $S$ (pai) & $N$ & $N$ & $N$ & 260 & EB inc & $E B$ inc & OK \\
\hline 16 & C & 2 & $D$ & $N$ & $N$ & $N$ & $N$ & $N$ & 375 & $E F$ & $E S$ & OK \\
\hline 17 & $C$ & 3 & $D$ & $N$ & $N$ & $N$ & $S$ (pai) & $S$ (pai) & 140 & $E F$ & $E F$ & $O K$ \\
\hline 18 & $C$ & 1 & $D$ & $N$ & $N$ & $N$ & $N$ & $N$ & 200 & EB inc & $E B$ inc & OK \\
\hline
\end{tabular}

A: Situação dos pais. S: solteiros, C: casados, D: desquitados; B: Gravidezes. D: desejadas, I: indesejadas; C: Tentativa de Aborto. N: não, S: sim; D: Antecedentes de Violência Familiar. N: não, S: sim; E: Antecedentes Psiquiátricos. N: não, S: sim; F: Renda familiar mensal em reais dividido pelo número de membros da família;

G: Escolaridade pai e mãe. EB: ensino básico ( $8^{a}$ série completa), EF: ensino fundamental ( $3^{\circ}$ colegial completo), ES: ensino superior, EB: inc: ensino básico incompleto; H: Condições de moradia. OK: casa de alvenaria + luz elétrica + esgoto encanado + água tratada.

Tabela 2 - Perfil socioeconômico familiar das 18 crianças.

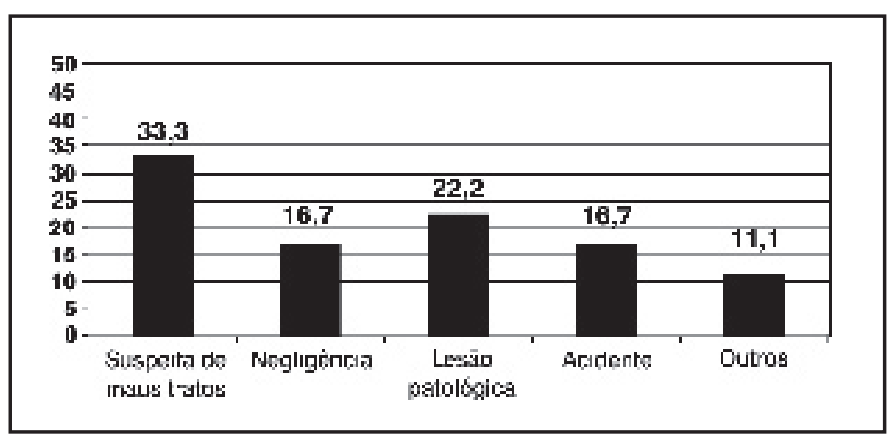

Fonte: SAME do Departamento de Ortopedia e Traumatologia da Santa Casa de Misericórdia de São Paulo.

Gráfico 2 - Distribuição quanto à causa das fraturas de fêmur nas 18 crianças, de acordo com escala modificada.

tramos que em quatro casos os pais apresentavam antecedente de violência familiar. Uso de drogas foi constatado em quatro famílias e etilismo em três. A renda familiar mensal por pessoa (renda familiar total / número de pessoas da família) variou de 86 a 500 reais. Oito pais e nove mães relataram ensino básico incompleto de escolaridade; apenas uma mãe refere ter completado o ensino superior. Em todos os casos reavaliados foi constatada a consolidação da fratura, confirmada mediante a radiografia simples.

Em relação aos pacientes que não foram contatados para a reavaliação $(n=17)$ verificamos que em nove casos o endereço e telefones contidos no prontuário médico não existiam; em quatro o endereço não correspondia ao do paciente/familiares/conhecidos; e em quatro os familiares mudaram-se sem fornecimento do novo local de moradia. Um paciente foi contatado e não compareceu para a reavaliação por estar residindo atualmente em outro Estado. Uma busca nos órgãos públicos quanto ao registro de novas internações e/ou óbito não ofereceu dado adicional.

\section{DISCUSSÃO}

Maus-tratos é definido, de acordo com o artigo 136 do Código Penal Brasileiro, como: "expor a perigo de vida ou saúde de pessoas sob sua autoridade, guarda ou vigilância, para fins de educação, ensino, tratamento ou custódia, quer privando-a de alimentação ou cuidados indispensáveis, quer sujeitando-a a trabalho excessivo ou inadequado, quer abusando de meios de correção ou disciplina"(10). As formas de maus-tratos são: física, sexual, psicológica ou negligência. Outras formas conhecidas são: Síndrome do Bebê Sacudido e Síndrome de Münchausen.

No Brasil, durante o período janeiro de 1998 a junho de 1999, foram registrados 1.169 casos de violência doméstica praticada contra crianças e adolescentes, sendo $22 \%$ crianças com até os três anos de idade. A mãe é indicada como principal agressora em $52 \%$ dos casos, e o pai em $27 \%$. A principal forma de violência denunciada foi física (65\% das ocorrências), sobrepondo-se com formas de violência psicológica (51\%) e negligência (49\%)(11).

Os sinais sugestivos de abuso infantil incluem a presença de múltiplas lesões agudas (equimose, hematoma, escoriações, mordidas, queimaduras e edemas de partes moles), história prévia de abuso, hematoma subdural, alteração comportamental, presença de múltiplas fraturas (principalmente fêmur, tíbia e úmero) e/ou fraturas em vários estágios de cicatrização; entretanto, fraturas isoladas ocorrem freqüentemente ${ }^{(12)}$.

Beals e Tufts ${ }^{(13)}$ relataram que $30 \%$ das crianças com menos de quatro anos de idade que haviam sofrido fratura de fêmur foram vítimas de abuso físico. Em crianças com menos de três anos, Dalton et $a^{(4)}$ encontraram um índice de $31 \%$ de ocorrências de maus tratos físicos. Anderson ${ }^{(7)}$ relatou um índice de $79 \%$ e $83 \%$ de suspeita de SMT como causa da fratura de fêmur em crianças com menos de dois anos e menos de 13 meses de idade, respectivamente. Outros estudos apontam uma prevalência de aproximadamente $60-85 \%$ de SMT em crianças com fratura de fêmur e idade inferior a um ano ${ }^{(5,6)}$. Entretanto, Blakemore et al(14), em 1996, em um estudo envolvendo 42 crianças de um a cinco anos, relataram somente um caso de abuso infantil, sendo o único achado na literatura com baixa relação entre fratura de fêmur em crianças em decorrência de maus tratos. Em nosso estudo observamos que em $50 \%$ das crianças reavaliadas há indícios sugestivos da SMT (maus tratos físicos e negligência) como desencadeante da fratura de fêmur, pois verificamos relatos discordantes, incompletos e inconsistentes em nove casos (Tabela 1). Entretanto, este é apenas um índice parcial, uma vez que, entre as 17 crianças que não foram reavaliadas por não comparecimento, pode haver um número maior de casos suspeitos ou mesmo 
confirmados de maus tratos, podendo ser este o motivo pelo qual os pais não responderem ao contato ou chamamento.

Crianças com menos de um ano de idade, ainda não deambulantes, são potencialmente as maiores vítimas de abuso; já as crianças mais velhas têm maior tendência e possibilidade de fuga do agressor ${ }^{(4,6)}$. Entre as crianças reavaliadas, observamos maior ocorrência de suspeita de SMT entre os 25 e 36 meses de idade, o que pode ser explicado pelo maior número de fratura dentro dessa faixa etária na população estudada (Gráfico 1). O predomínio de ocorrência de fraturas acidentais de fêmur em crianças do sexo masculino pode ser explicado pela maior exposição dos meninos às atividades recreacionais ${ }^{(15)}$.

O Código Penal prevê pena de multa aos médicos, professores ou responsáveis por estabelecimento de atenção à saúde e de educação que não comuniquem à autoridade competente casos de suspeita ou maus-tratos confirmados contra crianças ou adolescentes ${ }^{(10)}$. Dalton et al(4) mostraram que o médico ortopedista é o principal investigador (em número absoluto) de maus tratos físicos em crianças com fratura de fêmur, seguido pelo pediatra. A investigação de SMT em uma criança com fratura de fêmur deve ter início imediato e deve ser realizada por especialistas treinados. Histórias discordantes e inconsistentes contadas por diferentes pessoas ou mecanismos de trauma insuficientes para a ocorrência da lesão são achados altamente sugestivos de maus tratos em crianças. Uma das causas de fratura comumente referida pelos familiares é queda da cama ou de outras estruturas baixas, porém muitas vezes representa um mecanismo de trauma insuficiente para fratura de ossos longos ${ }^{(16-19)}$. Hennrikus et al $^{(16)}$ analisaram crianças com relato de queda da cama ou do colchão e observaram que 50\% dos menores de um ano e $5 \%$ das crianças entre um e cinco anos de idade eram potencialmente vítimas de abuso infantil. Nesse contexto, estudos controlados foram realizados e constataram relação direta de "relato de queda da cama" com abuso infantil ${ }^{(17-19)}$. Helfer et al ${ }^{(17)}$ avaliaram 81 crianças menores de cinco anos de idade que sofreram queda da cama enquanto internadas no hospital e encontraram apenas uma $(1,2 \%)$ ocorrência de fratura. Da mesma forma, Nimityongskul et al ${ }^{(18)}$ e Lyons et al ${ }^{(19)}$ reportaram a ocorrência de duas (2,6\% e 1\%, respectivamente) fraturas de ossos longos em crianças internadas em hospital e vítimas de queda da cama. Em nosso estudo observamos dois pacientes cuja causa relatada pelos pais foi queda da cama ou do colchão e suspeitamos que apenas um tivesse sido vítima de SMT. Outros 10 casos tiveram como causa relatada da lesão queda da própria altura, de rampas, de escadas baixas (até um metro), de cadeiras e do colo da mãe, sendo que em 6 crianças houve suspeita de SMT (Tabela 1). A investigação dos antecedentes familiares também pode auxiliar na identificação da causa da lesão. Charles e Shivas ${ }^{(20)}$ realizaram um levantamento da literatura relacionando gravidez e drogas ilícitas e observaram que 40\% (36/90 artigos) dos artigos revisados faziam analogia direta entre uso de drogas na gestação e abuso infantil. Em nosso estudo constatamos apenas um caso de fratura por negligência em que a mãe referia ser usuária de drogas ilícitas. Em outros dois casos constatamos que o pai era usuário de drogas, entretanto não houve associação direta com SMT. Quatro dos pacientes reavaliados em nosso estudo apresentaram antecedente de violência familiar por parte do pai, sendo 2 casos suspeitos de fratura infligida por maus tratos físicos e um caso decorrente de fratura por negligência.

Apesar de nossa amostra de pacientes pertencer a uma classe social baixa, a SMT não apresenta relação direta com o nível socioeconômico. Estudos em países desenvolvidos abrangendo populações com melhores condições sociais relatam alta prevalência de SMT em crianças vítimas de fratura de ossos longos ${ }^{(12,13)}$.

Um dos grandes problemas encontrados em relação ao abuso infantil é o efeito deletério que ocorre durante o crescimento e desenvolvimento do indivíduo, podendo levar a um déficit permanente da função cognitiva e/ou neuromotora. Prasad et al(8) demonstraram que crianças vítimas de abuso infantil apresentavam pior função cognitiva $(p<0,05)$, para habilidades motoras $(p<0,01)$ , de expressão $(p<0,001)$ e de recepção $(p<0,01)$ de linguagem durante seu crescimento. Profissionais da saúde têm, portanto, um compromisso social com a detecção e devem notificar casos suspeitos de SMT e estarem preparados para identificá-los.

\section{CONCLUSÃO}

Maus tratos constituem uma importante causa a ser investigada nos casos de fratura do fêmur em crianças com menos de três anos de idade, sendo o provável mecanismo responsável por metade das fraturas aqui estudadas. Os antecedentes familiares suspeitos e a identificação de mecanismos de trauma referidos pelos familiares insuficientes para a ocorrência da fratura representaram achados altamente sugestivos de maus tratos.

\section{REFERÊNCIAS BIBLIOGRÁFICAS}

1. Caffey J. Multiple fractures in the long bones of infants suffering from chronic subdural hematoma. Am J Roentgenol 1946; 56: 163-73.

2. Kempe $\mathrm{CH}$, Silverman FN, Steele BF, Droegemueller W, Silver HK. The battered child syndrome. JAMA 1962; 181: 105-112.

3. Rex C, Orth MS, Orth DNB, Orth MCh, Orth FRCS, Kay PR. Features of femoral fractures in nonaccidental injury. J Pediatr Orthop 2000; 20: 411-413.

4. Dalton HJ, Slovis T, Helfer RE, Comstock J, Scheurer S, Riolo S. Undiagnosed abuse in children younger than 3 years with femoral fracture. Am J Dis Child 1990; 144: 875-878.

5. Thomas SA, Rosenfield NS, Leventhal JM, Markowitz RI. Long-bone fractures in young children: distinguishing accidental injuries from child abuse. Pediatrics 1991; 88 (3): 471-476.

6. Schwend RM, Werth $C$, Johnston A. Femur shaft fractures in toddlers and young children: rarely from child abuse. J Pediatr Orthop 2000; 20: 475-481.

7. Anderson WA. The significance of femoral fractures in children. Ann Emerg Med $1982 ; 11: 174$.

8. Prasad MR, Kramer LA, Ewing-Cobbs L. Cognitive and neuroimaging findings in physically abused preschoolers. Arch Dis Child; 90: 82-85, 2005.

9. Loughrey CM, Preece MA, Green A. Sudden unexpected death in infancy (SUDI). J Clin Pathol; 58: 20-21, 2005.

10. Brasil. Presidência da República. Casa civil. Subchefia para Assuntos Jurídicos. Decreto-Lei 2848, de 07 de setembro de 1940. Disponível em: http://www. presidencia.gov.br/ccivil 03/Decreto-Lei/Del2848compilado.htm. Acessado em 2006 (Fev).

11. Associação Brasileira Multiprofissional de Proteção à Infância e à Adolescência
(ABRAPIA). Disponível em: http://www.abrapia.org.br/homepage/dados_sobre_violencia/dados_sobre_violencia_domestica.html

12. Santos LM, Stewart G, Meert K, Rosenberg NM. Soft tissue swelling with fractures: abuse versus nointentional. Pediatric Emergency Care 1995; 11 (4): 215216.

13. Beals RK, Tufts E. Fractured femur in infancy: the role of child abuse. J Pediatr Orthop 1983; 3:197.

14. Blackmore LC, Loder RT, Hensinger RN. Role of intentional abuse in children 1 to 5 years old with isolated femoral shaft fractures. J Pediatr Orthop 1996; 16 : 585-588.

15. Kotzias Neto A, Pierri CA Schneider A, Camisão GB, Oliver O. Avaliação dos resultados do tratamento das fraturas da diáfise femoral em crianças. Rev Bras Ortop 1998; 33 (1): 25-30.

16. Hennrikus WL, Shaw BA, Gerardi JÁ. Injuries when children reportedly fall from a bed or coach. Clinical Orthopaedics and Related Research; 407: 148-51, 2003.

17. Helfer RE, Slovis TL, Black M. Injuries resulting when small children fall out of bed. Pediatrics; 60: 533-35, 1977.

18. Nimityongskul P, Anderson LD. The likelihood of injuries when children fall out of bed. J Pediatr Orthop; 7: 184-86, 1987.

19. Lyons TJ, Oates RK. Falling out of bed: a relatively benign occurrence. Pediatrics; 92: 125-27, 1993.

20. Charles S, Shivas T. Mothers in the media: blamed and celebrated - an examination of drug abuse and multiple births. Pediatric Nursing, 28 (2): 142-145 March-April 2002. 